that a decline in patient Activities of Daily Living (ADL) function were associated with increases in time spent caregiving $(\mathrm{B}=.75 ; \mathrm{p}<.001)$, the likelihood and frequency of using home health services $(\mathrm{B}=.07$ and 1.6; $\mathrm{p}<.05$ and $<.001$, respectively), and depression symptom scores of the caregiver $(\mathrm{B}=.54 ; \mathrm{p}<.05)$. Increases in patient behavior problems were associated with increases in caregiver physical distress symptoms (dizziness, headaches, stomach and bowel problems) $(\mathrm{B}=$ $.03 ; \mathrm{p}<.01)$, caregiver visits to health care professionals $(\mathrm{B}=.15 ; \mathrm{p}<$ $.05)$, and use of counseling services $(\operatorname{Exp}(\mathrm{B})=1.21 ; \mathrm{p}<.05)$. Follow-up analyses showed that spouse caregivers were more likely than non-spouse caregivers to have an increase in depressive symptoms when reporting more patient ADL dysfunction $\left(\chi^{2}=3.86 ; \mathrm{p}<.05\right)$ and behavioral problems $\left(\chi^{2}=3.56 ; \mathrm{p}<.06\right)$. Conclusions: AD patient decline in ADL function and increases in inappropriate behaviors are associatd with increased caregiver depresion, physical distress and service use. Understanding the experience and behavior of continuous caregivers is important to developing methods for supporting this critical patient care resource.

\section{P3-465 CAREGIVING AND THE RELATIONSHIP TO CARE RECIPIENT'S COGNITIVE DECLINE}

Gwenith G. Fisher ${ }^{1}$, Brenda L. Plassman ${ }^{2}$, Jessica D. Faul ${ }^{1}$, Guy G. Potter ${ }^{2}$, John J. McArdle ${ }^{3}$, David R. Weir ${ }^{1}$, Mary A. M. Rogers ${ }^{4}$, Kenneth M. Langa ${ }^{4},{ }^{1}$ Institute for Social Research, University of Michigan, Ann Arbor, MI, USA; ${ }^{2}$ Duke University Medical Center, Durham, NC, USA; ${ }^{3}$ University of Southern California, Los Angeles, CA, USA; ${ }^{4}$ Department of Internal Medicine, University of Michigan, Ann Arbor, MI, USA. Contact e-mail: gwenithf@isr.umich.edu

Background: Caring for a cognitively impaired relative can be demanding and stressful. Research has also found that there are some rewards associated with the caregiving experience (e.g., feeling more useful, feeling closer to the care recipient, and feeling good about one's self). Little research has examined how caregivers' experiences are related to the patterns of cognitive decline. Objective: To examine how outcomes of caregivers' experiences in caring for a relative with dementia or cognitive impairment without dementia (CIND) are related to trajectories of cognitive decline in the care recipient. Methods: The present study examined longitudinal data from the Health and Retirement Study (HRS) and additional data collected in the Aging, Demographics, and Memory Study (ADAMS), in which in-home comprehensive dementia evaluations were conducted with a stratified sub-sample of 856 participants in the HRS, a representative population sample in the U.S. A consensus panel reviewed the results of these evaluations and assigned a diagnosis of dementia, CIND, or normal cognition. A knowledgeable informant completed a questionnaire about their caregiving experience; only information from family members who identified themselves as caregivers were included in this study. Cognitive trajectories of episodic memory (assessed by a word recall task administered up to 7 times over a period of up to 14 years) were examined. Results: Family caregivers of those diagnosed with dementia $(n=156)$ or cognitive impairment without dementia $(n=57)$ returned questionnaires (mean age $59.9 \mathrm{yrs} ; 51.6 \%$ with at least some college education; $72.3 \%$ women; $62 \%$ of caregivers were children, $22 \%$ spouses, and $16 \%$ other relatives). Latent growth curve models were calculated separately for individuals with dementia and those with CIND. Among caregivers of those with dementia (but not among those caring for individuals with CIND), higher levels of rewards were associated with lower levels of episodic memory and slower decline in memory scores over time $(p<.05)$. However, there was no relationship between perceived physical or emotional strain and cognitive decline. Conclusions: Caregiving for cognitively impaired individuals is associated with both strains and rewards. Important outcomes of the caregiver's experience (i.e. rewards) are related to trajectories of cognitive decline.

\section{P3-466 VALIDATION IN SPANISH OF THE SCREEN CAREGIVER BURDEN, SUBJECTIVE VERSION SCALE (SCB SB)}

Guadalupe Guerra-Silla, Óscar Rosas-Carrasco, Ulises Pérez-Zepeda, Mariano Montaña-Álvarez, Luis M. Gutiérrez-Robledo,

Jorge Reyes-Guerrero, Juan M. Villalpando-Berumen, Instituto Nacional de Ciencias Médicas y Nutrición, Mexico City, Mexico. Contact e-mail: lupina_guerra@hotmail.com

Background: Patients with dementia become dependants on caregivers, who are at risk of burdening because caregiving sometimes can be exhausting. There is no Spanish version of this scale (SCB SB) for Mexican population in Mexico. Methods: We performed a validation study on caregivers of dementia patients older than 60 years, who attended the memory clinic and already diagnosed as Alzheimer's disease, vascular, or mixed dementia. Caregivers answered the 25 -item screen caregiver burden scale, subjective version, and other related constructs such as BDI (Beck depression inventory), BAI (Beck anxiety inventory), MMSE (Mini mental state examination), QOL-AD (Quality of life in Alzheimer's disease), NPI-Q (Neuropsychiatric inventory questionnaire), SDI (Sleep disorders inventory), in two different times, 7 to 14 days apart. Results: Mean age of patients was 79.8 (SD: 8.17); mean of MMSE was 17.6 (SD: 6.16). $35.3 \%$ of patients suffered mixed dementia (Alzheimer's disease plus vascular); $31.4 \%$ vascular, and $26.5 \%$ Alzheimer's alone. $79.4 \%$ of caregivers were women; $20.6 \%$ were men. $51 \%$ of caregivers were patient's children; $32.4 \%$ were spouses; $7.8 \%$ were wage-earners. $68.3 \%$ of caregivers were married; $14.9 \%$ single; $9.9 \%$ divorced, and $5 \%$ widow. Mean hours per day of support given by caregivers: 14.7 ; mean days of support per week: 6.2. Mean score of SCB: 18.6 (SD: 13.62); median score of 15; mean score of BDI: 10.1 (SD: 7.50); mean score of BAI: 10 (SD: 7.93). We evaluated 104 caregiver-patients dyads, obtaining a Cronbach alpha of 0.85 for internal consistency; $\mathrm{CCI}$ of 0.76 for external consistency through the re-test procedure. Construct validity correlations, divergent and convergent between SCB and MMSE: $r_{s}=-0.25 p<0.028$; SCB and QOL-AD proxy: $r_{s}=-0.35 p<0.001 ;$ SCB and Lawton: $r_{s}=-0.19 p<0.044$; SCB and BDI: $r_{s}=0.52 p<0.001 ;$ SCB and BAI: $r_{s}=0.52 p<0.001$; SCB and NPI-B: $\mathrm{r}_{\mathrm{s}}=0.54 \mathrm{p}<0.001$; SCB and NPI-Q: $\mathrm{r}_{\mathrm{s}}=0.498 \mathrm{p}<0.001$; SCB and SDI: $r_{s}=0.387 \mathrm{p}<0.001$; SCB and SDI-B: $r_{s}=0.465 p<0.001$. These outcomes show a significant correlation between other constructs related to SCB SB. Conclusions: According to validity and reliability results it can be concluded that SCB SB is a scale that can be used to assess burden in caregivers of patients with dementia.

\section{P3-467 ALZHEIMER'S AND ABUSE: A 21ST CENTURY CULTURAL SWING}

Robin T. Hatcher, Hale County DHR, Greensboro, AL, USA. Contact e-mail: Robin.Hatcher@dhr.alabama.gov

Background: Alzheimer's Disease/Dementia has caused a very significant cultural swing within 21 st century social work due to the aging population within the United States. Social workers are being called upon more than ever to investigate possible cases of abuse, neglect and/or exploitation of victims suffering from dementia. In regards to these cases, many are a result of cruelty, but many more are a result of simple ignorance of the disease process on the part of the care provider. The care provider may not understand what is happening, so what starts out as an argument can end with an injury. In other cases, the alleged victim may not have been injured physically per a confrontation, but he or she might be totally ignored and an injury can result from the neglect. Lastly, as the victim's mental status declines and their dependence increases, their financial resources may go unprotected and the chance of their being financially exploited increases dramatically. Methods: Strong assessment skills are required to assess risks, capacity and needs of the victim and/or care provider so decisions can be made regarding future physical, mental, environmental and monetary care for the alleged victim. Social workers must be able to assess the situation, 